\section{Las enfermedades crónicas desde la mirada de los enfermos y los profesionales de la salud: un estudio cualitativo en México}

\author{
Chronic illness from the perspective of \\ patients and health professionals: a qualitative \\ study in Mexico
}

\author{
1 Universidad de \\ Guadalajara, Guadalajara, \\ México. \\ Correspondencia \\ F. J. Mercado-Martínez \\ Universidad de Guadalajara. \\ Jesús Galindo y Villa \\ 2941, Jardines de la Paz, \\ Guadalajara, México 44860. \\ francisco.mercado@cucs.udg.mx
}

\begin{abstract}
Chronic diseases are leading causes of morbidity, mortality, and increasing expenditures in numerous countries. However, little is known about how chronic diseases are perceived and managed by social actors. This article aims to compare the perspectives of health professionals and patients towards chronic diseases, besides analyzing the relationship between these two groups. A qualitative, multi-center study was conducted in three Mexican cities: Guadalajara, San Luis Potosí, and Mexico City. Participants included chronically ill individuals, physicians, and other health professionals from primary and secondary health care centers. Data collection used focus groups and interviews. The data were analyzed using discourse analysis. Participants' perceptions varied, from the medicalized view of physicians to that of patients focused on illness and the lifeworld. The participants agreed that there are unequal relationships between health professionals, families, and the chronically ill, but that relationships are more equal among the chronically ill themselves. The article includes by discussing various implications of the findings.
\end{abstract}

Chronic Disease; Health Services; Focus Groups
Francisco J. Mercado-Martínez ${ }^{1}$

Eduardo Hernández-Ibarra ${ }^{1}$

\section{Introducción}

Las enfermedades crónicas constituyen las primeras causas de morbi-mortalidad en numerosos países, incluyendo México 1. Tales enfermedades habrán de incrementarse en los próximos años, debido a factores como el impacto de la tecnología médica, el envejecimiento de la población y los nuevos estilos de vida y trabajo. El resultado será un mayor número de personas enfermas, una creciente presión sobre los servicios de salud y un incremento en los costos financieros, sociales, familiares y emocionales 2 .

Las enfermedades crónicas han sido objeto de atención de los profesionales de la salud. Estos han realizado incontables estudios clínicos, bioquímicos, farmacológicos, epidemiológicos y recientemente desde la biología molecular. Los criterios empleados son fundamentalmente de tipo biológico y cuantitativo, tales como la morbilidad y la mortalidad. Tal enfoque, sin embargo, no ha estado exento de críticas. Algunas de ellas por excluir las perspectivas de actores como los enfermos y sus familiares en torno a su origen, su evolución y manejo, su desinterés por la dimensión subjetiva y experiencial del padecimiento, su no reconocimiento de las prácticas no médicas empleadas para su prevención, manejo y rehabilitación, así como su carácter prescriptivo 3 .

En décadas recientes se han incrementado los estudios cuyo interés es la mirada de actores sociales sobre determinadas enfermedades 
crónicas y su atención 4,5. Una vertiente suele explorar la mirada de uno de los actores, sean los médicos, los enfermos, los familiares o los cuidadores 6,7,8. Otra compara las perspectivas de dos actores, sean las de médicos y enfermos, las de familiares y enfermos, o las de dos profesionales sanitarios 9,10,11. No obstante, muy pocos trabajos en nuestro medio se han interesado por este fenómeno y comparado la perspectiva de múltiples actores. El asunto reviste importancia porque estas enfermedades implican procesos complejos de naturaleza orgánica, emocional, social y económica, por lo cual explorar el punto de vista de un actor resulta insuficiente para comprender sus variadas facetas y efectos, así como para proponer programas de naturaleza más integral.

Este trabajo compara las perspectivas de dos grupos de profesionales de la salud y los enfermos sobre las enfermedades crónicas. Dos preguntas centrales guiaron la investigación: ¿cuáles son los tópicos de la enfermedad a los que suelen prestar atención estos grupos?, y iqué tipo de relaciones establecen estos actores en torno a dichas enfermedades crónicas?

\section{Metodología}

Este trabajo forma partedeun estudio más amplio sobre la perspectiva de actores sociales en torno a las enfermedades crónicas y su atención 12 . Una investigación cualitativa, multicéntrica, se llevó a cabo en tres ciudades de México: Guadalajara (1,6 millones de habitantes), San Luis Potosí (800.000) y México DF (15 millones). Seleccionamos un barrio en cada ciudad, donde habitara mayoritariamente población del sector popular y existiera un centro de la Secretaría de Salud. Estos centros y los hospitales de referencia brindan atención médica a población no asegurada y con pocos recursos.

Los sujetos seleccionados fueron médicos, profesionales sanitarios no médicos y personas con enfermedades crónicas. Los médicos trabajaban en centros de salud u hospitales; $43 \%$ eran residentes, $35 \%$ generalistas y $22 \%$ especialistas; más de la mitad eran hombres, y en promedio tenían 37 años de edad y nueve trabajando con enfermos crónicos. Los profesionales sanitarios no médicos eran casi en su totalidad mujeres (96\%), enfermeras $(49 \%)$, trabajadoras sociales $(22 \%)$, psicólogas (18\%) y auxiliares de enfermería y promotoras de salud. En promedio tenían 36,9 años de edad y 13,3 trabajando con enfermos crónicos. 67 personas enfermas participaron. Tenían 56,4 años en promedio y mayoritariamente eran mujeres (80\%). Un porcentaje semejante se dedicaba al hogar, no recibían remuneración ni estaban jubiladas. Los ingresos del otro $20 \%$ eran relativamente estables pero limitados, al ser obreras, trabajadoras del servicio doméstico, o dedicarse a lavar y planchar ropa o al cuidado de niños. Los hombres enfermos participantes no trabajaban, fuese por edad avanzada o como consecuencia de las enfermedades. Así, casi todos los enfermos dependían económicamente de sus familias; una cuarta parte tenía acceso a la seguridad social por periodos determinados al depender de la situación laboral de sus hijos. $58 \%$ de los enfermos padecían más de una enfermedad crónica, como diabetes, hipertensión, o artritis. Estas personas acudían a distintos tipos de atención médica, a la par del centro de salud asistían a la seguridad social, la medicina privada o con terapeutas tradicionales.

La información se obtuvo mediante grupos focales $13 \mathrm{y}$ entrevistas individuales. Las mismas estuvieron a cargo de dos investigadores en cada ciudad. Veinti-nueve grupos focales se formaron en total, diez en Guadalajara, ocho en ciudad de México y once en San Luis Potosí. Ocho correspondieron a médicos, diez a otros profesionales sanitarios y once a sujetos enfermos. Su número de integrantes osciló entre cinco y 14, lo cual dio un total de 194 participantes. La estrategia empleada para seleccionarlos y convocarlos fue similar, aunque haciendo adecuaciones. Los profesionales de la salud se seleccionaron al adscribirse a los centros de salud del barrio; los enfermos a partir del contacto previo con uno de ellos en el mismo centro de salud y el uso posterior del muestreo bola de nieve para los demás enfermos. Nuestro propósito fue incorporar a quienes desearan involucrarse voluntariamente en el estudio, una vez habiéndoles informado e invitado a participar. No fue posible implementar dicha estrategia en el caso de los profesionales al ser convocados por los directivos. Aun así, se les insistió en la naturaleza voluntaria de su participación. Las entrevistas a los enfermos se realizaron en casa de uno de ellos, aunque alguna se hizo en un centro asistencial o universitario.

Una guía de entrevista semi-estructurada se empleó en los grupos focales. Dicha guía constaba de cinco apartados: introducción, experiencia del padecimiento, atención a la enfermedad, educación sanitaria, y síntesis/clausura. Este trabajo incluye básicamente la información del segundo apartado. Un investigador fungió como coordinador y otro como co-coordinador de los grupos focales. Las entrevistas fueron grabadas y transcritas por una persona, utilizando indicaciones para la trascripción. El análisis de la información se basó en el análisis crítico del discurso siguiendo la propuesta de Fairclough 14. Tal aná- 
lisis implicó explorar la función textual donde se capta lo que la gente dice, los temas emergentes y su magnitud; la función relacional ocupada de las interacciones y los términos en que se dan, y la función ideacional que explora las creencias e ideas de los participantes.

Al final del estudio se presentaron los resultados a los participantes en sus respectivos espacios. Además de enriquecer el trabajo con nueva información, dos situaciones se presentaron. Los participantes agradecieron la presentación de los resultados comentando ser la primera ocasión que los recibían; a la vez, cuestionaron la utilidad de la investigación, argumentando las dificultades para resolver los problemas. Aún así nos solicitaron presentar los resultados a quienes pudieran apoyar en su solución. En esta investigación se cumplieron los principios éticos contenidos en la Declaración de Helsinki.

\section{Resultados}

\section{La perspectiva de los sujetos enfermos}

Las personas enfermas aluden básicamente a tres enfermedades crónicas, la diabetes, la hipertensión y la artritis o "reumas", como suelen llamarla. Hacen referencia a tales condiciones a través de un conjunto de temas ordenados jerárquicamente. Tales temas suelen ubicarse en el contexto de los problemas que enfrentan en su vida diaria.

El discurso de estas personas prioriza varias manifestaciones orgánicas. Entre ellas, dolor de cabeza, pérdida de peso, hambre y sed intensa, debilidad, somnolencia, deseos frecuentes de orinar, dolor de piernas, pérdida de la vista y mareos. Un enfermo de Guadalajara y otro de San Luis dan cuenta de tal variedad cuando aluden a sus padecimientos: “... he sentido (...) dolor de cerebro, mucho sueño, mucho cansancio, mucha sed, y a veces muchas ganas de ir al baño...”, “... [me] duelen mucho los pies, veo muy borroso y [tengo] mucho sueño, [ando con] cansancio, como si anduviera débil, donde quiera [uno] se quiere dormir...".

Las manifestaciones y trastornos emocionales derivadas de su(s) padecimiento(s) se mencionan en un siguiente plano. Destacan la tristeza, la desesperación, los deseos de llorar, la irritabilidad, la depresión y su poca tolerancia ante situaciones adversas. Un enfermo de San Luis describía su situación al verse limitado en sus labores cotidianas “...le da a uno coraje, le da a uno ansias (...) me da coraje [de] que ya uno no puede (...) no tienes ganas de hacer [nada], le da a uno como nervios (...) y se deprime uno mucho...".
Varios enfermos reconocían los efectos de tales manifestaciones en su vida laboral en términos de que les imposibilitan trabajar, no son los mismos y se vuelven dependientes de quien los cuidan o ayuda a realizar sus labores. Una mujer de Guadalajara describe tal situación “...ya no puede uno desempeñar su quehacer (...) como lo desempeñaba antes de estar enfermo, [antes] trabajaba uno a gusto y ya ahora no; las enfermedades ya no lo dejan a uno [trabajar], empieza uno a sentir cansancio, aquel desatino. Mejor me siento y el quehacer ahí se queda parado...".

Los sujetos enfermos se refieren a continuación al tratamiento y al manejo de su enfermedad. A la par de las consultas con los médicos, el ejercicio y las dietas, también las infusiones, los remedios caseros y las pomadas juegan un papel importante en su tratamiento. En un siguiente plano aluden a las causas por las cuales aparecieron sus padecimientos crónicos. Los sustos, las sorpresas, los corajes y las preocupaciones se combinan sin dificultades con los factores hereditarios. La mayoría trata este asunto en términos semejantes, siempre destacando el factor emocional como una causa

En menor grado hacen referencia al control de las enfermedades y las causas por las cuales se dan las complicaciones. Para casi todos, los problemas de su vida diaria imposibilitan el control de su enfermedad. Ello se ejemplifica cuando una participante señala “...una persona diabética mejora si es bien tratada y comprendida; te friega más [cuando hay un] problema (...) [por ello] te sube la azúcar, la presión y te sube todo y el medicamento no te hace nada...".

Según los enfermos participantes, la presencia divina tiene una influencia decisiva en sus enfermedades sea porque las provoca, les ayuda a sobrellevarlas o a curarlas. Entre las incontables alusiones a tal presencia divina, comentarios de los participantes ilustran el tenor del asunto "pos tú [Dios] sabrás, así me tienes y así yo estoy contenta", "es un consejo que Dios mandó, para que uno se enmiende un poquito...”, “cuando Dios quiera [me curo]", "esperemos en Dios [que mejore]" y "gracias a Dios me bajó la azúcar".

Las personas enfermas, por otra parte, tienen presentes las relaciones que establecen con los profesionales de la salud, sus familias y otras personas enfermas debido a la enfermedad, así como la forma como se dan tales relaciones: desiguales e insatisfactorias. En su discurso aparece en primer lugar su relación con los médicos, en términos de no ser escuchados, no dárles la atención necesaria, no hablarles y recibir un trato poco humanitario. Un enfermo de Guadalajara y otro de México coinciden en tal apreciación “... sería mucho mejor que [los médicos] aparte de 
ser profesionales, fueran humanitarios (...) pueden evitar muchos errores...”, “....mi doctora no me hace caso (...)le digo que me duelen mis piernas y se me adormecen. Dice que es un problema de fondo y de ahí no la saco. Escribe y escribe sus notitas y nada más me da el medicamento para la presión...". La relación que entablan con sus familiares también es objeto de mención, pero aludiendo básicamente a los conflictos generados por la enfermedad. Entre otros refieren aquellos derivados de la irritabilidad del enfermo, por ser dependientes y no participar en los quehaceres domésticos. Por ello son objeto de regaños y relegados. Otra mujer de Guadalajara trasmite la impresión de muchas otras cuando dice “...[mi familia] me relega, hasta los hijos le dicen a uno ‘ya estás como viejita, ya estás ahí acostada!'. Mi pareja se molesta, mis hijos me ven como algo que enfada...". Por otra parte, la relación y el trato que establecen con las enfermeras se dan en términos semejantes al ser objeto de regaños. Una enferma de la ciudad de México describe una situación que menciona frecuentemente “...si usted va enfermo [al centro de salud] y está con dolor y les pregunta [a las enfermeras] '¿no me toca ya?' responden 'iespérese!' (...)Y aunque uno este ahí llorando, ellas bien tranquilas...". Las relaciones que entablan con otras personas enfermas, sean los grupos de autoayuda, clubes de enfermos o vecinos también ocupan un lugar central en su discurso. Pero a diferencia de las anteriores, consideran estas relaciones en términos positivos, dado que suelen recibir apoyo a la par que son satisfactorias. Por ejemplo, cuando saben de alguien con una enfermedad crónica lo invitan a los grupos, le recomiendan algún médico o centro de salud, socializan el uso de determinados remedios o le dan consejos.

La perspectiva de los profesionales sanitarios no médicos

Varias enfermedades crónicas, sobre todo la diabetes, la hipertensión arterial, la insuficiencia renal y la artritis aparecen en el discurso de estos profesionales; pero destacan la diabetes y la hipertensión. Estos profesionales también se refieren a varios tópicos en orden jerárquico. El panorama que ofrecen es complejo, mientras aluden a múltiples aspectos de la enfermedad a la par profundizan en ellos.

Sobresalen en su discurso las manifestaciones y efectos de las enfermedades crónicas. Entre ellas destacan, en orden jerárquico, las emocionales, las familiares, las orgánicas, las económicas, las laborales y las sociales. Para estos profesionales, la depresión, la autoestima baja, la no aceptación de la enfermedad, la ansiedad, el llan- to, la angustia, el miedo, el aislamiento, la irritabilidad y el coraje ocurren frecuentemente entre las personas enfermas. Enfermeras de Guadalajara y México destacan tales manifestaciones en términos semejantes “...emocionalmente tienen muchos cambios (...) principalmente los diabéticos: son corajudos...”, “...llegan [los enfermos] a veces en estados depresivos (...) tenemos que ayudarlos a que salgan y que acepten y asimilen su enfermedad...". Entre los efectos familiares más mencionados a causa de estas enfermedades se encuentran su dependencia, la desintegración de la familia, la molestia de los familiares, e incluso las enfermedades entre los familiares debido al cuidado. Una enfermera comenta sobre el particular “...hay casos en que el paciente fallece, después [el familiar] continúa atendiéndose [en el centro de salud] pero con otra patología diferente o con una enfermedad crónica (...) Cuando fallece el paciente, el familiar continúa atendiéndose, después de una larga jornada de estrés y cansancio [debido al cuidado]...”. A continuación estas profesionales aluden a problemas orgánicos como la pérdida de peso, cansancio, sed constante, poliuria, mareos y vómitos. Estas enfermeras también tienen presente los efectos económicos y laborales de tales trastornos. Según ellas, los enfermos dejan de trabajar debido a la pérdida de sus capacidades por su enfermedad. Ello incrementa sus necesidades económicas, en tanto sus gastos son mayores y sus ingresos menores, lo cual se complejiza al ser objeto de estigma y discriminación. Una profesional de San Luis Potosí explica tal situación “...los pacientes en edad laboral se enfrentan a tener que dejar de trabajar porque ya no tienen las mismas capacidades (...) se sienten rechazados por la sociedad, [la enfermedad] es un tabú, o sea, si eres diabético iya!....".

Estas profesionales aluden en menor grado al control de tales condiciones. Según ellas, los enfermos no están controlados por no ser constantes en sus dietas y medicamentos, no disponer de recursos económicos para adquirir alimentos y medicinas, además de hacer cambios frecuentes al tratamiento. Un profesional de Guadalajara comenta sobre la circularidad del cumplimiento y el control haciendo eco de los comentarios del grupo “...cuando los pacientes se sienten bien [dejan de tomar el medicamento], y cuando no lo toman se vuelven a sentir mal; [luego] lo vuelven a tomar (...) hacen muchos cambios de los medicamentos...".

A lo largo de las entrevistas, estas profesionales se refieren a otros temas, pero asignándoles menor importancia. Por ejemplo, para muchas la herencia, los hábitos alimenticios y la obesidad son las causas de las enfermedades crónicas, particularmente de la diabetes y la hipertensión. 
También destacan la insuficiencia renal, el pie diabético, las amputaciones y la pérdida de las extremidades como las complicaciones habituales de los enfermos crónicos. Por ello insisten en las dietas, los medicamentos y el ejercicio físico como medidas básicas del tratamiento y el control.

Las relaciones entre los profesionales de la salud y los sujetos enfermos son vistas por estas profesionales de forma diferente a lo reportado por los enfermos. En primer lugar consideran que su relación con los enfermos es cercana. Según sus narraciones, suelen escuchar a los pacientes, les ayudan y los tratan de forma cálida y amable. Una enfermera de Guadalajara valora el asunto de la forma siguiente: “...nosotras, más que los médicos, somos las que estamos constantemente con los pacientes, (...) quienes están dando constantemente la cara...”. Estas profesionales también tienen presente la relación de los enfermos con sus familiares, destacando el mal trato de que suelen ser objeto, ya que la familia no los tolera, los aísla, deja de apoyarlos y los abandona. Un profesional de la salud de Guadalajara comenta lacónicamente “...la familia abandona en muchos casos a los pacientes; el esposo o la pareja se enfadan de estar con la carga de la enfermedad y los abandonan". La relación entre los médicos y los enfermos también es objeto de su escrutinio. Desde su perspectiva, los médicos son fríos en su trato a los enfermos, además de ser restrictivos, brindarles una atención deficiente, y olvidarse de que son personas. Una enfermera describe escuetamente tal relación “...el área médica los relega, no les hacen [caso], ven mucho el síntoma y se olvidan de la persona, [los médicos] están viendo el tumor, están viendo la tuberculosis, pero se olvidan de la persona...”. En coincidencia con lo expresado por los enfermos, tanto enfermeras como psicólogas mencionan que la relación entre los enfermos es buena, máxime cuando pertenecen a grupos o clubes de autoayuda. Una enfermera dice convincentemente “...ellos mismos se ayudan tanto que se sienten bien (...) hacen ejercicio, comparten sus experiencias, conviven, se invitan e informan de los laboratorios y se dan pláticas...".

\section{La perspectiva de los médicos}

El discurso de los médicos se centra básicamente en tres enfermedades, la diabetes, la hipertensión y la artritis, destacando las dos primeras como referentes. A diferencia de los anteriores, el eje de su discurso gira en torno a las enfermedades, luego a los servicios de salud y finalmente a los pacientes.

El discurso de estos médicos prioriza las manifestaciones emocionales, incluso sobre las orgánicas y el tratamiento, mientras que los efectos familiares, sociales y laborales de la enfermedad pasan prácticamente desapercibidos. La ansiedad, la depresión, la angustia, el llanto y el rechazo de la enfermedad son los trastornos que mencionan en primer lugar. Un médico de Guadalajara destaca tales manifestaciones “...he visto que su autoestima [de los enfermos] baja bastante cuando se les confirma una enfermedad crónica; algunos la rechazan y otros no lo creen...". Paralelo a ello aparecen reiteradamente en sus discursos diversas manifestaciones y efectos orgánicos, como las cefaleas, los mareos, el evento vascular cerebral y la mala circulación.

Estos médicos aluden, aunque dándole menos importancia, al control metabólico y las causas de estas enfermedades. Según ellos, los enfermos no siguen los tratamientos, se sienten desanimados ante la falta de medicamentos y lo inadecuado de las dietas. Explican tal situación bajo el argumento de que los enfermos saben poco, desconocen o son indiferentes ante sus enfermedades. Un profesional de Guadalajara expresa con desánimo “...mucha gente se vuelve indiferente, se acostumbra a su enfermedad y a vivir descontrolada...". Un factor adicional que incide en el control de la enfermedad, según estos médicos, es el estado emocional de los enfermos. Uno de ellos lo refiere de forma breve, pero contundente “...los pacientes, mientras más problemas depresivos y más cuadros de angustia presenten, más se enferman...”.

Para estos médicos, la causa de las enfermedades crónicas, particularmente de la diabetes e hipertensión, es hereditaria a lo que se añade la obesidad y el sedentarismo a las cuales la denominan como factores de riesgo. Por ello, los medicamentos, la dieta y el ejercicio siguen siendo las medidas más idóneas para su manejo.

Las relaciones que entablan diferentes actores en torno a estas enfermedades son vistas por estos médicos de forma sui generis. En primer lugar resaltan su relación con los enfermos, pero reconociendo ciertas particularidades. Según ellos, los enfermos consideran maltrato cuando los médicos no los miran ni los revisan. Un médico de Guadalajara intenta explicar el asunto pero sin profundizar"...si nosotros no los vemos, para ellos es una falta de atención, falta de respeto, yo creo que de ahí implica ese maltrato que ellos captan...". Otros reconocen que los mismos médicos se limitan a dar indicaciones a los pacientes, pero dan sus razones tal como lo menciona un médico de San Luis Potosí “...preferimos darles indicaciones muy concretas. Pocas veces les decimos, ‘qué dudas tienen?'...”. Según ellos, tal situación obedece a que las autoridades les limitan el tiempo de la consulta. 
Estos médicos también hacen referencia a la relación entre los enfermos y su familia. Según ellos, la familia no los apoya, ni los escucha, ni participa en su manejo. Un médico de la ciudad de México expone brevemente el asunto “...al enfermo lo dejan solo, le preparan su comida aparte, a veces lo dejan que viva aparte, no participan de su problemática...". En el discurso de estos médicos también aparece la relación que establecen con los directivos de los centros u hospitales. Según algunos, tales directivos sólo se preocupan de los asuntos administrativos, más no de los médicos, tal como lo expone escuetamente un médico de Guadalajara “...el directivo (...) está en su oficina, hace sus cosas administrativas, acaba y se va...". Así se quejan de ellos argumentando que les limitan el tiempo y se desinteresan por su capacitación. Por último, los médicos también notan diferencias entre los enfermos que asisten a los grupos de autoayuda y los clubes de enfermos respecto a quienes sólo acuden a consulta; los primeros se interesan más en seguir el tratamiento, muestran mayor disponibilidad, se apoyan entre ellos e intercambian ideas y recetas. "En el club de diabéticos llega una señora y puede preparar [comida] de una manera y de otra forma. Todo esto los enriquece porque entre ellos [los enfermos] se andan pidiendo las recetas...", comenta un médico de San Luis Potosí sobre tal relación.

\section{Discusión}

Este estudio ha explorado la perspectiva de tres actores sociales sobre las enfermedades crónicas y sobre las relaciones sociales que se establecen en torno a las mismas. Entre los hallazgos centrales destaca la diversidad de posiciones e intereses de los participantes en cuanto a variadas facetas de las entidades crónicas y su manejo; también sobresalen distintas relaciones que establecen los actores involucrados en la trama de la enfermedad.

Los resultados del trabajo muestran similitudes y diferencias en las perspectivas de los sujetos enfermos, los médicos y el personal sanitario no médico, tal como se ha reportado en otros trabajos 15. Cada actor destaca diferentes dimensiones de la enfermedad y su manejo, y cada uno hace referencia a fenómenos distintos; los médicos aluden a las enfermedades, la atención médica y los servicios de salud; mientras que los sujetos enfermos a sus molestias, sus padecimientos y al manejo en el marco de los problemas de su vida diaria. El personal de salud no médico combina elementos de los unos y los otros. El reconocimiento de estas diferencias puede entenderse como una situación conflictiva, pero también como un elemento donde se entrevén alternativas para mejorar la atención médica. En este contexto, el reconocimiento de posturas diferentes no significa inferioridad, indiferencia o ignorancia, ni lleva a la descalificación, la exclusión o el dominio 16, como ocurrió en algún momento de la atención a la enfermedad. Al igual que como lo han reportado otros autores 17, los médicos participantes en este estudio reproducen una concepción biomédica en donde a una entidad mórbida previamente definida se le asigna un tratamiento convencional. Los sujetos enfermos, por su parte, enfatizan múltiples manifestaciones y dificultades que enfrentan en el marco de su vida diaria. En este contexto, adquieren relevancia las dificultades a las que hacen referencia los enfermos, tales como las derivadas de lo laboral y sus ingresos y que inciden en la trayectoria del padecimiento. En un país como México, en donde casi la mitad de la población vive en la pobreza, estas indicaciones de los enfermos no pueden ser pasadas por alto, dadas sus implicaciones en el manejo de la enfermedad crónica.

Según ciertos autores, los profesionales de la salud comparten una visión homogénea de la enfermedad y su tratamiento, al reproducir una imagen biomédica y medicalizada 18 . De acuerdo a nuestros resultados, tal visión dista de ajustarse a la realidad, sobre todo al compararse la perspectiva de médicos y enfermeras. No contamos con una explicación sobre tales diferencias, pero nos parece que además de sus distintas formaciones, ello pudiera obedecer al contacto que establecen cotidianamente estas enfermeras a lo largo de tantos años con los sujetos enfermos y sus familias, sobre todo en el primer nivel de atención. Otro factor a considerar es la mayor movilidad de los médicos respecto a otros profesionales sanitarios, en tanto suelen durar menos tiempo laborando en estos centros de salud.

Pero ipor qué un tratamiento biomédico por parte de los médicos ante padecimientos complejos, máxime cuando reconocen las manifestaciones emocionales y físicas, así como dificultades financieras, de los servicios de salud, e incluso de los enfermos? Tenemos dos hipótesis de trabajo al respecto. Por un lado, ello se puede explicar en función de la entrevista empleada para la obtención de los datos en este estudio, esto es, los grupos focales, la cual fue aplicada en instalaciones sanitarias. En ese marco, la información proporcionada por los médicos puede estar más vinculada a un deber ser y a la información social y políticamente correcta que a sus propias concepciones y prácticas sobre la enfermedad crónica y su manejo. Algunos médicos, por ejemplo, proporcionaron información distinta fuera 
de la entrevista grupal, bajo el argumento de que podrían ser sancionados si hicieran públicamente referencias a determinados posturas y problemas. La segunda hipótesis es que los médicos pueden estar percibiendo los trastornos emocionales, pero distan de ofrecer tratamiento o solución alguna al no estar capacitados para ello. Una indicación en este sentido es la recomendación que suelen hacer de contratar psicólogos para mejorar la atención médica.

Incontables estudios se han realizado sobre la relación médico paciente o entre diversos grupos o sistemas de atención ${ }^{19}$. Sin embargo, pocos trabajos centran su atención en las relaciones que establecen los actores en torno a enfermedades crónicas. En este estudio los participantes coinciden en la existencia de relaciones desiguales entre médicos, enfermeras, familiares y enfermos. Estos últimos son objeto de rechazo, regaño, aislamiento o reconvención por parte de todos ellos. El asunto reviste importancia al evidenciarse mecanismos de desigualdad en torno a problemas sanitarios concretos, lo cual puede afectar el manejo y el control de estas enfermedades. Otros estudios habrán de explorar tal situación. Una hipótesis de trabajo es que nuestros resultados podrían deberse al hecho de que se trata de enfermos del sector popular que presentan al menos varias "desventajas sociales": ser pobres, dependientes, mayoritariamente mujeres, en edad adulta o ancianas y viviendo con enfermedades crónicas. Sería pertinente explorar tales relaciones con otros enfermos, pero sin desventajas, por ejemplo, en varones, jóvenes y de sectores medios y altos.

Los participantes del estudio coinciden en la existencia de relaciones más igualitarias entre los sujetos enfermos. Ello en términos de que se apoyan entre sí, se recomiendan o sugieren médicos y tratamientos, se retroalimentan y dan consejos. Este asunto ha sido objeto de reflexión, sobre todo en trabajos que plantean las ventajas de los grupos de autoayuda, ayuda mutua y las asociaciones de enfermos 20 . No conocemos trabajo alguno realizado en Latinoamérica en esta dirección teniendo presentes las enfermedades crónicas. Sin embargo, el tema habrá de explorarse con detenimiento porque expone una veta que podría ser esencial para transformar los servicios de salud. Por ejemplo, en caso de comprobarse estos resultados, los servicios de salud podrían pasar a ser organizados y evaluados cada vez más por la población y particularmente por los enfermos, los cuidadores y sus familiares.

Una voz faltante en este estudio es la de los cuidadores y los familiares. Tal como lo han reportado otros autores 21 , la perspectiva de estos actores es vital para comprender mejor el ám- bito doméstico como parte de la atención a la enfermedad crónica y que suele pasar desapercibida por las políticas y los servicios de salud. De hecho, llama la atención la visión negativa que tienen los participantes sobre la familia y la falta de reconocimiento a sus contribuciones en el cuidado de los enfermos crónicos. Será cuestión de escuchar la voz y los argumentos de quienes dedican parte importante de su vida, tiempo y energías al cuidado de sus parientes enfermos.

Varias implicaciones derivan de lo expuesto. Frente a un modelo médico terapéutico vigente, un número creciente de voces abogan por un modelo más incluyente y participativo. En este contexto, los resultados encontrados en este estudio pueden ser de utilidad en varios sentidos. Por ejemplo, las carencias financieras que hemos reportado en otro trabajo 22 constituyen un ejemplo de cómo distintos puntos de vista pueden ser útiles para evidenciar la complejidad del tratamiento de estas enfermedades. Cada grupo alude a dichas carencias pero desde distintos ángulos. Mientras uno reporta las del sistema de salud, otro se refiere a las del centro sanitario u hospital, y otro más a las del ámbito familiar y doméstico o a los efectos de la enfermedad en los ingresos, máxime que se trata de población sin seguridad social. El manejo de los enfermos crónicos, según estos hallazgos, implica enfrentar situaciones complejas que van más allá de la prescripción de fármacos.

Este trabajo se centra en las perspectivas de profesionales de la salud y sujetos con enfermedades crónicas. El mismo se preocupa por el mundo de las concepciones, las representaciones y las imágenes, más que el de las prácticas, las estructuras y los procesos. Los resultados aquí expuestos, por ende, no exploran el mundo de lo que se hace y del contexto que rodea a los participantes. Aún así, ello no implica dejar de reconocer los conflictos en el interior de los programas sanitarios al prevalecer puntos de vista contrapuestos sobre el mismo fenómeno. En este contexto no deja de ser una posición reduccionista basar las propuestas sanitarias en el punto de vista de uno de los actores, esto es, a la de los médicos. Las prácticas y programas de salud habrán de transformarse en la medida que también incorporen las voces y discursos de estos actores involucrados. Frente a un modelo médico caracterizado, entre otras cosas, por imponer el punto de vista de los médicos, los hallazgos nos llevan a proponer una lectura de los fenómenos y procesos sanitarios basada en el contrapunto, es decir, a narrar las historias a partir de distintas voces 23 , o parafraseando a Bourdieu 24, a abandonar el punto de vista único, central y dominante en beneficio de la pluralidad de puntos de 
vista coexistentes y a veces rivales. Los resultados expuestos en este trabajo apuntan a la existencia de miradas sobre las enfermedades crónicas no coincidentes y en algunos planos contradictorias. Será cuestión de debate y negociación llegar a acuerdos sobre tales diferencias, máxime cuando las mismas podrían estar fundamentadas en diferencias de clase, género y profesiones.

Nuestros resultados podrían ser objeto de discusión dadas las estrategias utilizadas. Las entrevistas a grupos focales, por ejemplo, se han convertido en técnicas sobreempleadas en la investigación en salud. Su bajo costo y resultados inmediatos han sido razones poderosas que favorecen su utilización. Sin embargo, sus desventajas son notorias, tal como lo encontramos en el estudio en curso. Uno de los presupuestos de los grupos focales consiste en que los participantes no deben conocerse 25 ; sin embargo, circunstancias como la del presente trabajo hacen imposible cumplir dicha condición, dado que se trata de comunidades pequeñas o de profesionales que laboran en unidades médicas donde todo el personal se conoce. Por ello, la información obtenida en este contexto podría estar limitada al menos por dos razones: por un discurso plagado de información considerada socialmente aceptable o deseable, sobre todo por los profesionales de la salud, y por una visión que excluye cualquier referente crítico a determinadas posturas o prácticas de los individuos, grupos o servicios de salud. Por ello, convendría utilizar estrategias que impliquen al menos la triangulación de métodos.

\section{Resumen}

Las enfermedades crónicas constituyen las primeras causas de morbi-mortalidad e implican costos crecientes para los servicios sanitarios en numerosos países. Sin embargo, poco se conoce la forma como diversos grupos las perciben y enfrentan. Este trabajo compara las perspectivas de dos tipos de profesionales sanitarios y población enferma sobre tales enfermedades y sobre las relaciones existentes entre los actores involucrados. Un estudio cualitativo, multicéntrico, se realizó en tres ciudades de México: Guadalajara, San Luis Potosí y México DF. Participaron sujetos con enfermedades crónicas, médicos y profesionales sanitarios no médicos. La información se obtuvo mediante grupos focales y entrevistas individuales, analizándose mediante análisis del discurso. La perspectiva de los participantes sobre tales enfermedades varía entre la mirada medicalizada de los médicos, frente a la de los sujetos enfermos centrada en los padecimientos y la vida diaria. Los participantes coinciden en la existencia de relaciones desiguales entre los trabajadores de la salud, la familia y las personas enfermas, así como en relaciones más igualitarias entre los enfermos. Se discuten múltiples implicaciones de los resultados.

Enfermedades Crónicas; Servicios de Salud; Grupos Focales

\section{Colaboradores}

F. J. Mercado-Martínez participó en el planeamiento y diseño del estudio, la obtención de la información y el análisis de los datos, así como en la redacción del artículo. E. Hernández-Ibarra participó en la búsqueda, recuperación y análisis de la bibliografía, así como en el manejo y análisis de los datos. También colaboró en la redacción del artículo.

\section{Agradecimientos}

A Leticia Robles por sus comentarios valiosos; a Brenda Trigueros por su apoyo en la edición. A los participantes por su colaboración inestimable y a los comentaristas anónimos por sus estimulantes observaciones. 


\section{Referencias}

1. Secretaría de Salud. Información epidemiológica. http://www.dgepi.salud.gob.mx (accedido el 04/ Ene/2006).

2. Beaglehole R, Yach D. Globalisation and the prevention and control of non-communicable diseases: the neglected chronic diseases of adults. Lancet 2003; 362:903-8.

3. Michie S, Miles J, Weinman J. Patient-centredness in chronic illness: what is it and does it matter? Patient Educ Couns 2002; 51:197-206.

4. Conrad P. Qualitative research on chronic illness: commentary on methods and conceptual development. Soc Sci Med 1990: 30:1257-63.

5. Fernández J, Santos MA. Evaluación cualitativa de programas de educación para la salud. Una experiencia hospitalaria. Málaga: Ediciones Aljibe; 1992.

6. Calnan MW. The patient's perspective. Int J Technol Assess Health Care 1998; 14:247-54.

7. Sullivan M. The new subjective medicine: taking the patient's point of view on health care and health. Soc Sci Med 2003; 56:1595-604.

8. Robles L. Género, pobreza y cuidado: la experiencia de mujeres cuidadoras pobres urbanas. In: Salgado N, Wong R, organizadores. Envejeciendo en la pobreza. Género, salud y calidad de vida. Cuernavaca: Instituto Nacional de Salud Pública; 2003. p. 123-51.

9. Barry CA. Multiple realities in the study of medical consultations. Qual Health Res 2002; 12:1093-111.

10. Bennett CL, Chapman G, Elstein AS, Knight SJ, Nadler RB, Sharifi R, et al. A comparison of perspectives on prostate cancer: analysis of utility assessments of patients and physicians. Eur Urol 1997; 32(3 Suppl):86-8.

11. Lawler FH, Viviani N. Patient and physician perspectives regarding treatment of diabetes: compliance with practice guidelines. J Fam Pract 1997; 44:369-73.

12. Mercado-Martínez FJ, Alcántara ECG, Tejada LMA, Lara N, Sánchez A. O atendimento médico aos doentes crônicos no México: quais as diferenças entre a perspectiva dos médicos, das enfermeiras e das pessoas doentes? In: Bosi MLM, Mercado FJ, organizadores. Pesquisa qualitativa de serviços de saúde. Petrópolis: Editora Vozes; 2004. p. 363-400.
13. Morgan DL. The focus groups guidebook. Thousand Oaks: Sage Publications; 2000.

14. Fairclough N. Analyzing discourse. London: Routledge; 2003.

15. Burman ME. Multiples perspectives on being uninsured and barriers to health coverage in a rural western state. J Health Care Poor Underserved 2006; 17:625-40

16. Parisi A. Diferencias. In: Salas R, organizador. Pensamiento crítico latinoamericano. Conceptos fundamentales. v. I. Santiago: Ediciones UCSH; 2005. p. 189-99.

17. Hunt L, Arar N. An analytical framework for contrasting patient and provider views of the process of chronic disease management. Med Anthropol Q 2001; 15:347-67.

18. Kleiman A. Patients and healers in the context of culture. An exploration of the borderland between anthropology, medicine and psychiatry. Berkeley: University of California Press; 1980.

19. Menéndez E. Modelos de atención de los padecimientos: de exclusiones teóricas y articulaciones prácticas. Ciênc Saúde Coletiva 2003; 8:185-207.

20. Drevdahl D. Coming to voice: the power of emancipatory community interventions. ANS Adv Nurs Sci 1995; 18:13-28.

21. Robles L. La pobreza urbana: ¿Cómo sobrevivir enfermo y pobre? In: Arias P, Woo O, organizadores. Nuevos espacios y formas de vida en el campo y la ciudad. Guadalajara: Universidad de Guadalajara; en prensa.

22. Mercado FJ, Trigueros BI. Living poor and chronically sick in the third world. A qualitative study in Mexico. In: Cook K, Gilbert K, editors. Problematizing marginalization: perspectives on physical, social and mental health. Frenchs Forest: Pearson; 2006. p. 156-76.

23. Said EW. La pluma y la espada. México DF: Editorial Siglo XXI; 2001.

24. Bourdieu P. La miseria del mundo. México DF: FCE; 1999.

25. Morgan DL. Focus groups as qualitative research. Thousand Oaks: Sage Publications; 1997.

Recibido el 21/Feb/2006

Versión final presentada el 01/Feb/2007

Aprobado el 05/Abr/2007 